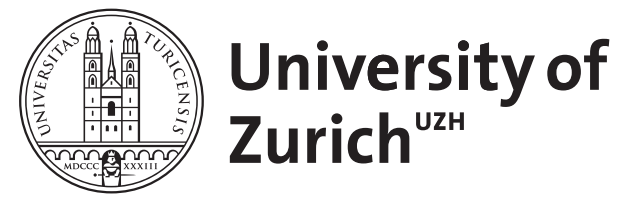

Zurich Open Repository and Archive

University of Zurich

University Library

Strickhofstrasse 39

CH-8057 Zurich

www.zora.uzh.ch

Year: 1994

Rhétorique de la perte. L'exemple de la mort d'Isabelle de Bourbon (1465)

Kiening, Christian

Posted at the Zurich Open Repository and Archive, University of Zurich

ZORA URL: https://doi.org/10.5167/uzh-123767

Journal Article

Published Version

Originally published at:

Kiening, Christian (1994). Rhétorique de la perte. L'exemple de la mort d'Isabelle de Bourbon (1465). Médiévales: Langue, Textes, Histoire, 13(27):15-24. 


\section{Rhétorique de la perte. L'exemple de la mort d'Isabelle de Bourbon (1465)}

In: Médiévales, N²7, 1994. pp. 15-24.

\section{Abstract}

The Rhetoric of Loss - The fifteenth century evidences, in a variety of diverse ways, to a growing interest in affective phenomenons and their literary and figurative representations. The author has chosen, from among the vast number of laments in which a veritable phenomenology of mourning is developped, three texts which refer to the same event : the death of Isabel of Bourbon (1465). These texts throw light on the relationship between historical fact and literary imagination as well as on the (anthropological) tension between mourning and consolation.

Citer ce document / Cite this document :

Kiening Christian. Rhétorique de la perte. L'exemple de la mort d'Isabelle de Bourbon (1465). In: Médiévales, N²7, 1994. pp. 15-24.

doi : 10.3406/medi.1994.1307

http://www.persee.fr/web/revues/home/prescript/article/medi_0751-2708_1994_num_13_27_1307 
Médiévales 27, automne 1994, pp. 15-24

Christian KIENING

\section{RHÉTORIQUE DE LA PERTE \\ L'EXEMPLE DE LA MORT D'ISABELLE DE BOURBON (1465)}

La position du Moyen Âge chrétien face au deuil était, on le sait, ambivalente. Un système sotériologique comme le système chrétien où la mort ne possédait qu'un caractère de transitus, où le temporel était transcendé par l'éternel, avait du mal à intégrer le phénomène d'un deuil intramondain, phénomène néanmoins anthropologique ${ }^{1}$. Ainsi voit-on côte à côte des relativisations ou des réglementations du deuil, commençant avec l'interdit encore modeste de l'apôtre Paul (I Thess. 4,13 sq.), et des acceptations d'un dolor iustus (par exemple chez saint Augustin). Il faut cependant attendre l'établissement et le développement des littératures vernaculaires dès le XII' siècle - et avec ceux-ci des conceptions pas originellement chrétiennes - pour voir les expressions du deuil et de la douleur s'étendre et s'enrichir ${ }^{2}$. Cette " réhabilitation du deuil ", qui ne cherchait pas la confrontation directe avec le modèle chrétien de la consolatio, l'influençait pourtant profondément. Les dimensions d'un long processus de transformation se manifestent clairement au cours du XVe siècle où apparaissent des traités, sous la plume même des clercs, qui justifient les expressions du deuil et de la douleur en tant qu'articulations propres à la nature humaine (par exemple Guilelmus Savonensis, An mortui lugendi sunt an non), où la représentation des pleurants se fait cons-

1. P. von Moos, Consolatio: Studien zur mittellateinischen Trostliteratur über den Tod und zum Problem der christlichen Trauer, 4 vol., Munich, 1971/72, p. 129 sq. (contient aussi une bibliographie abondante) ; U. MENNECKE-HAUSTEIN, Luthers Trostbriefe, Gütersloh, 1989, p. 99 sq. ; G.W. MCCLURE, Sorrow and Consolation in Italian Humanism, Princeton, 1991.

2. G. Duby, "Réflexions sur la douleur physique au Moyen Âge ", dans Mâle Moyen Âge, Paris, 1988, pp. 203-209; voir aussi les actes du colloque II dolore e la morte nella spiritualità dei secoli XII e XIII, Todi, 1967. 
tituante des monuments funéraires ${ }^{3}$, où complaintes en prose et en vers, déplorations funèbres et épitaphes forment des modes littéraires auxquels presque tous les auteurs participent ${ }^{4}$.

Je retiens ici le genre de la complainte qui me semble apte à illustrer les possibilités et les problèmes d'une description historique du deuil. Étant enracinée dans le planctus latin, la complainte a trouvé dans les langues vernaculaires, en particulier dans les langues romanes, des formes spécifiques témoignant d'une évolution autonome du genre. Ce qui est caractéristique de cette évolution - que l'on peut suivre d'Eustache Deschamps et de Christine de Pizan jusqu'aux deux premières générations des "grands rhétoriqueurs " ${ }^{5}$ - c'est l'augmentation de l'ampleur et surtout la croissante subtilité thématique aussi bien que formelle : jeux de mots, mètres complexes, effets visionnaires et allégorisations s'inscrivent dans une poétique de l'intertextualité et de l'autoréférence qui touche au maniérisme ${ }^{6}$. En même temps, l'interaction de l'émotion et de la rhétorique, constitutive pour toutes sortes des complaintes, prend des dimensions nouvelles. On y joue avec la dialectique de proximité et distance. On ne déplore pas la mort de sa propre épouse, mais la mort de personnes de haut rang et on s'adresse ainsi à un public auprès de la cour, cercle dont l'auteur lui-même fait partie. On se réfère à un consensus présumé par lequel l'auteur devient porte-parole de la communauté, mais qui réduit aussi les libertés de l'inventio poétique.

Ainsi, les complaintes se situent au point d'intersection de deux lignes : la ligne diachronique, qui définit la position d'un texte en relation avec l'évolution du genre, et la ligne synchronique, qui joint un événement historique à son adaptation littéraire. Les complaintes fournissent des renseignements historiques qui complètent souvent les rapports des chroniques, surtout sur le plan de l'émotion, mais elles doivent être aperçues dans leurs formes spécifiquement littéraires. Pour mieux saisir les interférences entre l'histoire et la littérature on peut considérer les cas où plusieurs complaintes partent du même événement, du même décès. C'est le cas, parmi d'autres, de la mort d'Isabelle de Bourbon dont (au moins) trois complaintes enrichissent non seulement les récits plutôt maigres des chroniqueurs, mais manifestent, semble-t-il, un tournant du genre : " Au fil des années 1460 ", constate Claude Thiry, «le concert des voix poétiques s'amplifie pour

3. É. MÃle, L'Art religieux de la fin du Moyen Âge en France, Paris, 1949 , $5^{e}$ éd., p. $418 \mathrm{sq}$. ; cf. aussi le catalogue de l'exposition Les pleurants dans l'art du Moyen Âge, Dijon, 1971.

4. C. Thiry, La Plainte funèbre, Turnhout, 1978 (avec une bibliographie commentee) ; voir aussi V. B. RICHMOND, Laments for the Dead in Medieval Narrative, New York, 1966.

5. C. Martineau-Genieys, Le thème de la mort dans la poésie française de 1450 à 1550, Paris, 1978, p. 295 sq.

6. Cf. P. ZuMTHOR, Le masque et la lumière : Le siècle des grands rhétoriqueurs, Paris, 1978. 
chanter la gloire. La Mort reste haïssable, mais elle peut être dominée [...] ; de plus en plus fréquemment, le deuil est balayé par l'apothéose, les larmes séchées au souffle de la Renommée ". ${ }^{7}$ La perspective synchronique confirme-t-elle cette description?

Lorsqu'Isabelle de Bourbon mourut de la tuberculose le 26 septembre 1465 à l'âge de 31 ans, ce ne fut pas un grand événement pour la politique contemporaine - à part le fait qu'elle n'avait donné à son mari qu'une seule fille, Marie (de Bourgogne), et pas d'héritier mâle ${ }^{8}$. La plupart des chroniqueurs n'enregistrèrent le décès, qui fut précédé de longs mois de maladie, que laconiquement ${ }^{9}$. Pour eux l'événement le plus intéressant de la vie d'Isabelle fut son mariage avec Charles le Téméraire, en 1454. Ce mariage politique, décidé sans l'accord de son fils par le duc Philippe le Bon et arrangé en cachette, se changea inopinément en une liaison d'amour - " tellement", comme le dit Jacques Du Clercq dans ses Mémoires, "qu'il n'estoit point sceu que puis que le comte l'euist espousée ». Il est bien possible que cette aura du couple amoureux ait inspiré les auteurs des complaintes, car aucun n'oublie de mentionner l'amour entre Isabelle et Charles - d'autant plus que Charles, occupé à des négociations politiques après sa victoire dans la bataille de Montlhéry, ne put assister au décès de son épous ${ }^{10}$. Amé de Montgesoie, dans sa complainte, fait contraster fortement la « triumphant victoire " de Charles et le malheur d'" avoir perdu du siecle la meilleur » (v. 44/48) ". Et une chanson anonyme exprime le regret d'Isabelle " sans mesure et sans compas / Pour son mary qui n'y fut pas / Qu'elle avoit amé de cœur bon " (v. 36-38) ${ }^{12}$.

Cette chanson de six couplets (48 vers), en annonçant un " piteux recors ", invite explicitement les auditeurs à la compassion et conduit successivement jusqu'aux derniers moments de la mourante. Les deux premiers tiers du texte décrivent le deuil de tous les gens liés à Isabelle et la perte douloureuse qui touche toute la société, l'Église aussi bien que les " pauvres gens" (v. 25 sq.). Le dernier tiers focalise le cen-

7. C. ThIRY, " De la mort marâtse à la mort vaincue : attitudes devant la mort dans la déploration funèbre française ", dans Death in the Middle Ages, H. BRAEIT et W. VerbeKE éd., Louvain, 1982, pp. 239-257 (cit. p. 250 f.).

8. Sur Isabelle cf. L. Hommel, Marie de Bourgogne ou le Grand Héritage, Bruxelles, 1945, pp. 59-66 ; G.H. Dumont, Marie de Bourgogne, Paris, 1982.

9. Cf. Olivier de la Marche, Mémoires, [...], H. Beaune et J. Arbaumont éd., Paris, 1833-36, t. III, p. 24. La description la plus détaillée est donnée par JacQues DU CleRCQ, Mémoires, dans Chroniques d'Enguerrand de Monstrelet, J. A. BUCHON éd., Paris, 1826/27, Supp., t. XV, p. 59.

10. Sur Charles of. P. Contamine, Des Pouvoirs en France, Paris, 1992, pp. 87-98.

11. T. Walton, "Les poèmes d'Amé de Montgesoie ", Medium Aevum, t. 2, 1933, p. 133 (texte pp. 21-28) ; sur Amé, cf. l'article dans le Dictionnaire des Lettres Françaises: Le Moyen Age, G. HASENOHR et M. ZINK dir., Paris, 1992, p. 55 sq.

12. Chants historiques et populaires du temps de Charles VII et de Louis XI [..], M. Le: RouX DE Lincy éd., Paris, 1857, p. 77 sq. 
tre des événements, en montrant comment Isabelle prend congé de sa famille (« son lignage de Bourbon »; v. 40), comment elle recommande ses parents et finalement sa fille (qui n'était pas présente) à Dieu. La fin de la chanson rejoint son début, où l'auteur avait demandé la miséricorde divine pour Isabelle.

Tous les éléments de cette complainte - la lamentation, l'apologie, le regret et la prière - sont bien connus; ils se subordonnent assez clairement à l'intention de présenter la fin de " madame de Charolloix " de façon authentique et émouvante. Avec sa mort bien organisée dans le cercle de la famille, Isabelle offre l'exemple d'une bonne mort chrétienne, l'exemple d'un acte harmonieux de mourir - en conformité avec sa vie qui fut consacrée «Sans chesser [...] traittier l'accord et la paix " (v. 23 sq.). La concordance profonde entre les sentiments de la mourante et ceux des conjoints survivants s'insère parfaitement dans le paradigme " de l'humaine mortalité » (v. 8).

Ceci est aussi la perspective centrale de la complainte d'Amé de Montgesoie qui donne, en effet, une description beaucoup plus complexe des circonstances du trépas. Amé, valet de chambre d'Isabelle, qui fut présent à la mort de sa noble dame, enrichit le tableau par un nombre considérable de détails historiques concernant par exemple le "grief mal qui trois mois lui dura " (v. 59), les réactions des gens en face d'une " perte tresmaleureuse " (v. 127) ou le " fin drap d'or bordé de velours noir " (v. 257) qui couvrait le corps ${ }^{13}$. Il présente un « long reportage rimé », d'une prétention littéraire plus élevée, lequel débute par une forte attaque contre la Mort et s'élargit enfin vers des réflexions plus globales sur la loi de la nature et la fragilité humaine.

Avec son attaque contre la Mort, Amé reprend une figure rhétorique qui était déjà établie de cette façon par Eustache Deschamps et Alain Chartier, et dont les antécédents se laissent poursuivre jusqu'aux œuvres de Chrétien de Troyes et aux planh provençaux ${ }^{14}$. Souvent, chez Amé aussi, l'élaboration figurative de la personnification reste pâle ; le seul élément concret qui apparaît dans presque tous les textes est le «dart " de la Mort. La figure exprime pourtant un phénomène psychologique essentiel ${ }^{15}: d^{\prime}$ 'une part la déviation de la douleur en agression contre une puissance insaisissable, d'autre part la substitution paradoxale d'un Toi perdu par un Toi " jamais existant " qui représente la " contre-force » par excellence, l'« Ennemie des cuvres de Nature » (v. 3), principe de la destruction, cause de

13. Cf. T. Walton, loc. cit., p. 32, qui cite un passage des livres des comptes (« A Jehan Dulon, pour avoir fait et refait par deux fois ung pale de drap d'or cramoisy tresriche, bordé de velours noir $\|)$.

14. E. SCHULZE-BUSACKER, « La complainte des morts dans la littérature occitane " dans Le sentiment de la mort au Moyen Âge, C. Sutro éd., Montréal, 1979, pp. 231-248.

15. Cf. H. StUввE, Formen der Trauer: Eine kulturanthropologische Untersuchung, Berlin, 1985, p. 100 sq. ("Trauerzerstörung und -agression »). 
la souffrance. Ce double caractère de l'apostrophe de la Mort se manifeste clairement chez Amé par l'entremêlement des éléments agressifs, plaintifs et glorifiants.

Après l'exposition " du grant dueil excessif » (v. 31), en particulier celui du malheureux époux, Amé conduit ses lecteurs directement dans " la maison borbonnoise " (v. 52) pour mettre en scène les derniers moments de la comtesse de Charollais. Son récit confirme ce que l'on sait, par la chanson anonyme, sur les recommandations d'Isabelle et il ajoute très précisément comment la mourante reçut les sacrements, fit lire un ars moriendi, baisa la croix et rendit son esprit. Les réactions sont unanimes : "Sospirs cuisans, langoreuses complainctes, / Cris angoisseux, effusions de lermes " (v. 118 sq.). Eux aussi, " ces gens doulans ", attaquent « la Mort maugré » (v. 132), plaignent la perte d'un exemple et d'un miroir de vertu, expriment une douleur "sans recueil, / Sans faire fais digne de hault louer " (v. 215f.). Amé, en reprenant ces lamentations, les rend plus générales, faisant référence à la nature entière d'une façon qui rappelle les poèmes sur la mort d'Orphée ; il imagine que la Terre transformerait "son vert " (v. 197), que les éléments rejetteraient tout ce « qui a plaisir sortisse " (v. 203), que les oiseaux changeraient leurs chants mélodieux " en coy frenesieux " (v. 205). Mais la complainte contient déjà des points de la consolation : Amé évoque le fait qu'aucune lamentation, aucune douleur ne peut ranimer un corps transi, que tous les hommes tomberont dans les mains de la mort. C'est justement en tournant le regard vers la nature, qui semble être violemment blessée par la mort, que l'auteur découvre l'inéluctable régularité de ses lois (" sa labeur ordonnee ", v. 233), qu'il reconnaît le principe bien connu de la fragilité humaine : l'homme n'est pas autre chose qu'une " fleur qui passe sans duree" (v. 236), qu'une ombre diminuante. En conséquence, Amé souligne que de 1' " excellent corps d'Isabelle de Bourbon " (v. 10), couvert par un beau linceul, ne reste plus qu'un cadavre " pour estre habandonné es vers » (v. 257). Et il s'insère ainsi dans une longue tradition chrétienne du contemptus mundi : comme les plaintes ne servent à rien, Amé conseille de se rendre à Dieu et il demande finalement de ne pas prier seulement pour l'âme d'Isabelle mais aussi pour la sienne : pour qu'« amé de moult je soye " (v. 270).

Ce rébus en forme de prière finale (que l'on retrouve aussi dans la deuxième œuvre d'Amé, le Pas de la Mort) signale que le panegyricus, chantant la gloire d'une personne morte ou vivante, vise également à l'augmentation de la gloire de l'auteur. Ceci ne remet pas en question la sincérité des sentiments, car la complainte d'Amé de Montgesoie exprime aussi bien le deuil des parents survivants que sa propre douleur, son " angoisseulx desplaisir " (v. 222). Mais le poète joue le double rôle du plaignant et du consolateur. C'est pour cela qu'il tente, en élaborant largement le récit du trépas, en rassemblant des faits et des émotions, en insérant directement les réactions des parti- 
cipants, de généraliser parallèlement la confrontation avec la mort. Le modèle, présent en arrière-plan, est encore celui de l'ars moriendi. Mais il ne résout plus tous les problèmes qui se posent face à la mort, qui naissent de l'exigence d'authenticité. On rencontre, dans ce drame de la perte situé au "Parc de Dueil » (v. 9), une ambivalence latente entre l'accroissement rhétorique du deuil et la nécessité chrétienne de l'abolir. La fin du texte étant encore réservée au retour vers Dieu, la consolation semble plutôt s'effectuer à travers le regard sur la nature en tant que principe de vie et de mort.

Pour pouvoir estimer la représentativité de cette perspective sur la mort, il faut prendre en considération la troisième complainte, celle de Pierre Michault, le texte le plus long (512 vers) et sans doute le plus orienté vers des modèles littéraires ${ }^{16}$. Michault, bien qu'œuvrant auprès de la cour d'Isabelle, ne fut pas présent lors de sa mort. Il n'y a pas de raison de douter de l'indication du début de sa complainte qu'il eût séjourné dans ce temps-là « En ung païs loingtainnement distant " (v. 1), probablement auprès de Charles le Téméraire ${ }^{17}$. Le fait que Michault ne pouvait connaître la mort de la comtesse que de seconde main - par un " raport " (v. 15) et peutêtre par la complainte d'Amé qu'il a utilisée, semble-t-il, dans la partie finale de sa complainte - change profondément la forme du récit. La perspective y est plus personnelle, elle exclut presque totalement la présence d'un public qui était si important pour les deux autres complaintes pour témoigner du caractère universel de la douleur. Au lieu du récit historique : le raisonnement personnel.

La nouvelle de la mort d'Isabelle, considérée également en tant que résultat de l'action puissante de la Mort (" par sa rudesse / et par son dart ", v. 17 sq.), évoque la réflexion. Pour Michault, l'action de la Mort représente une " énormité » qui n'est pensable que si l'on admet que « Dieu n'en tient compt ou Raison n'y voit goute » (v. 39). Il ne veut pas accepter la " difformité " de la création divine, l'agression contre la nature, contre le principe de la vertu : "Ou il convient loy estable interrompre / ou nouvelle creation reprendre / autre moyen sur ce ne puis entendre " (v. 62-64). Les alternatives sont, bien sûr, rhétoriques, mais elles prouvent le déplaisir de supporter la mort de celle qui était "en tout honneur de pure vertu sainte" (v. 50). L'auteur, absorbé dans ses pensées, se retire dans un verger sans pouvoir oublier. Brusquement il lui arrive - « en estasie / par trop penser et par ymaginer " (v. 78 sq.) - de voir apparaître deux dames bien différentes devant lui, figurant son conflit intérieur : Vertu et la Mort. Ensuite, il se développe en 28 couplets une altercation qui,

16. Pierre Michault, OEuvres poétiques, B. Folkart éd., Paris, 1980, pp. 143-169 (texte critique dans l'article du même auteur : " Perspectives médiévales sur la mort ", Le Moyen Français, t. 4, 1979, pp. 29-74).

17. En novembre 1466 Charles le retient comme " secrétaire signant " (cf. l'article du Dictionnaire des Lettres Françaises, op. cit., p. 1186). 
caractéristique pour les altercations médiévales, oppose plutôt des arguments et des concepts que de véritables figures. Vertu accuse d'abord la Mort d'être dure, fausse et chétive. Celle-ci se montre surprise, ne veut pas s'y reconnaître et demande des précisions. Vertu se nomme et $s$ 'explique, en variant surtout les lamentations sur la perte prématurée de sa " noble creature " (v. 158), sur la "disjuncture et divorce / de deux vrays cuers " (v. 187 sq.). La position de la Mort est, somme toute, plus nuancée. Elle insiste sur son droit, son activité aveugle, son indifférence face aux vices aussi bien qu'aux vertus. Elle répond aux plaintes navrées avec des phrases générales, surtout stoïques, contente voire imbue d'elle-même. Et c'est la Mort, enfin, qui se tourne vers la gloire de la défunte, qui souligne que c'est par son action même qu'Isabelle s'inscrit dans une mémoire éternelle (" en cronique ou hystoire "), qu'elle reçoit la "gloire de Paradis » (v. 289-296). C'est la Mort qui, en accentuant la fin " tresvertueuse ", la bonne mort chrétienne de la comtesse, donne le branle à une modification importante du dialogue : sa dernière réplique se termine par la sommation, adressée à Vertu, de raconter les derniers moments, les dernières phrases de la mourante.

Vertu donne, après une brève introduction, la parole à Isabelle. Par cette astuce narrative d'une double diffraction dans le récit, Michault arrive à rendre présentes les circonstances du décès sans avoir été lui-même présent. On trouve dans les paroles finales d'Isabelle une sorte de combinaison de deux formes narratives des autres complaintes : rapportant, comme la chanson anonyme, en discours direct les prières de la "gisant ", Michault suit en même temps l'élaboration plus détaillée de l'acte de mourir qu'avait donnée Amé. Les prières elles-mêmes se rattachent à celles, fortement répandues, des livres d'heures et des livres de bien mourir. Isabelle, en insistant continuellement sur la pauvreté de la créature, implore la miséricorde du Dieu, créateur et rédempteur, l'aide de Notre Dame et des saints. Ellc s'adresse enfin à son époux absent et prend congé de sa famille. Tous les éléments de cette dernière phase sont en parfait accord avec la description d'Amé : la lecture de l'ars moriendi, le souhait des intercessions après la mort, le baisement de la croix, etc. C'est encore l'exemplarité d'une mort qui s'y manifeste et qui est soulignée de nouveau dans les deux derniers couplets par la bouche de Vertu.

Or, la complainte de Pierre Michault doit se comprendre, en rapport avec celle d'Amé, en tant que conception complémentaire aussi bien que contrastante. Michault n'offre pas un deuxième reportage complet des circonstances de la mort d'Isabelle, mais il fait de nécessité - son absence à l'événement - vertu. Il transfère toute l'action dans un espace clos qui, vide de presque tous les aspects d'un public de cour, reflète la réciprocité de l'intérieur et de l'extérieur : justement au centre de l'altercation, la Mort apprend à Vertu qu'elle avait déjà entendu parler de son aveuglement " en ung traictié par cest auteur dité " (la Dance aux aveugles; v. 210). Ainsi Michault suit le modèle 
littéraire du récit allégorique rêvé où l'individu songeant est lui-même impliqué, mais il caractérise son apparition expressément - et de manière plus différente que dans la Dance aux aveugles - comme une " ymagination en estasie ", pas comme un rêve. Quoique l'attitude de Michault face à cette apparition, jugée en tant qu'aberration des sens (v. 80 ; " la dure frenesye ", v. 77), reste critique, les termes dont il use manifestent cependant l'exigence d'une plus grande véridicité de cette "ymagination" que celle d'un rêve. Comme Michault ne pouvait pas dépasser la facticité du récit de son prédécesseur, il chercha une nouvelle authenticité qui se fonde sur les faits connus, mais qui les insère dans un processus d'expérience.

On peut y trouver la raison du changement de registre assez frappant entre le dialogue et l'exemplum ${ }^{18}$. L'altercation entre la Mort et Vertu ne résout pas le problème principal : l'acceptation de la mort d'Isabelle. Bien que la Mort ait les meilleurs arguments, Vertu, obstinée, ne cesse pas ses complaintes, car l'argumentation n'est pas suffisante pour faire comprendre cet événement jugé inconcevable ; ce qui est nécessaire, c'est la représentation du trépas. C'est elle qui confirme ce qui n'était connu d'abord que par ouï-dire, et c'est par elle que la mort en tant que figure insaisissable est transformée en mort en tant qu'événement historique. Il est ainsi significatif que le seul élément que Michault ajoute sur le plan des faits soit le dernier mot de la mourante : "Credo " (v. 496). Ce mot peut être conçu également comme l'aveu de l'auteur d'avoir accepté ce qui lui était au début inacceptable. Seulement à la fin de la vision, se retrouvant seul dans le verger, il est capable de constater : « j'apperceux par ce que lors j'oÿ / que Mort avoit de la dame jö̈ " (v. 507 sq.).

Michault s'approche ainsi du processus d'expérience, du modèle dialectique de la Consolatio Philosophiae de Boèce (qui est cité par Vertu), sans l'atteindre. Il ne scrute pas le raisonnement philosophique sur l'existence humaine, mais la prolongation de la vertu au-delà de la mort. Pourtant il a saisi, à travers la double diffraction du récit, la problématique de l'individu face à l'événement historique et la tension entre les faits " réels " et la connaissance personnelle; et il a senti, plutôt inconsciemment, la nécessité du " travail du deuil » qui s'exprime de plus en plus souvent dans des textes littéraires et autobiographiques du XVc siècle ${ }^{19}$.

En regardant l'ensemble des trois complaintes, on ne trouve pas de divergences essentielles sur le plan des événements, mais on y trouve, sur le plan de l'imaginaire, différents modèles littéraires de la consolation qui méritent l'attention. Le déroulement du trépas est aussi assuré que la douleur profonde que la mort d'Isabelle pro-

18. Pierre Michault, op. cil., p. 150.

19. Un exemple extraordinaire en est donné par le marchand florentin Giovanni di Pagolo Morelli ("Ricordi ") à cause de la mort de son fils (1406) ; voir R.C. TREXLER, Public Life in Renaissance Florence, New York, 1980, pp. 161-186. 
voqua dans tout son entourage. On ressentit fortement la perte de la noble dame, toutefois sans tomber dans le désespoir. Après des mois de maladie, sa mort ne fut pas une grande surprise. Elle était " apprivoisée " par son caractère public et, surtout, par l'absence de Charles le Téméraire. Il se peut que les complaintes d'Amé et de Michault s'adressèrent implicitement, entre autres, à Charles pour lui présenter des récits plus détaillés et plus nuancés d'un événement qui devait lui tenir fortement à cœur; et il faut ajouter qu'un certain changement d'humeur de sa part était parfois mis en rapport avec la mort de son épouse aimée ${ }^{20}$. Celle-ci donna par sa mort l'exemple d'un décès parfaitement chrétien tel qu'il était défini par les arts de bien mourir : "Avoir conscience de sa fin prochaine, avoir du temps pour recevoir le saint viatique, avoir autour de soi assemblés clercs et laïcs, parents et amis, telles sont les conditions de la meilleure mort "; et il semble que les auteurs, avec une grande unanimité, se sont saisis de la chance de pouvoir parler d'une mort qui était - comme peut-être peu d'autres - conforme au modèle ${ }^{21}$. Mais ils n'en restèrent pas là. En exposant tout un spectre d'éléments de la douleur - tels que le choc, l'agression, l'identification avec la défunte, etc. - ils ont aussi présenté ce que la psychologie moderne a découvert en tant que phases essentielles du deuil humain.

Il y a d'autres convergences plutôt littéraires parmi les complaintes - comme celle de l'accentuation de la gloire ou celle de l'exigence de l'authenticité - qui pourraient s'intégrer dans la tendance que l'on a appelée "la victoire sur la mort " (cf. note 7). Or, la situation est plus complexe. La perspective synchronique montre que les auteurs ont tenté de créer l'authenticité soit par l'extériorisation, soit par l'intériorisation des événements. Les implications de ces deux approches se manifestent clairement une génération plus tard, dans les complaintes de Jean Molinet et Olivier de la Marche lors de la mort de la seule fille d'Isabelle, Marie de Bourgogne ${ }^{22}$. Jean Molinet, commençant sa complainte par une longue description de la ville de Bruges au jour de l'enterrement et de ses difficultés à trouver un logement, la termine par un dialogue - en latin - entre le malheureux époux, Maximilien, et la défunte, dialogue qui ranime momentanément le Toi perdu et qui réalise une dernière présence de l'absente ( lant il n'est riens impossible ", v. 400). Olivier de la Marche, quant à lui, après avoir offert dans une première partie plutôt traditionnelle les circonstances du trépas, clame enfin sa propre douleur et développe une altercation - aperçue " en une dorme-veille " - entre

20. Cf. L. HOMMEl, op. cit., p. 66.

21. R. Chartier, "Les arts de mourir, 1450-1600", Annales ESC, t. 31, 1976, pp. $51-70$, en particulier p. 66.

22. Jean Molinet, Faictz et dictz, N. Dupire éd., t. I, Paris, 1937, pp. 162-180; Olivier DE LA MARCHE, "Complainte sur la Mort de Madame Marie de Bourgogne ", dans Recueil des chansons, poèmes et pièces en vers français relatifs au Pays-bas, t. III, Bruxelles, 1878, pp. 25-38. 
l'œil, qui ne veut pas croire ce qu'il voit, et l'âme qui confirme et console. Les tendances de l'extériorisation et de l'intériorisation apparaissent ainsi inextricablement liées. C'est dans l'exigence d'authenticité que la documentation, de plus en plus détaillée, des événements extérieurs d'un décès rejoint la présentation, de plus en plus nuancée, d'un conflit intérieur. Mais, en insérant la mort dans un entremêlement de circonstances et de sentiments, on lui rend aussi une nouvelle forme de présence. C'est elle que l'on retrouve dans la dialectique presque paradoxale de deux attitudes face à la mort : d'une part, sur le plan idéologique, la négation ou la diminution de la mort par la renommée et la gloire, d'autre part, sur le plan anthropologique, l'acceptation du phénomène du deuil. Les conséquences de cette dialectique, touchant profondément à l'efficacité du modèle chrétien de la consolation, apparaissent déjà en arrière-plan de la bonne mort dont parlent les complaintes pour Isabelle de Bourbon. Et c'était déjà l'apôtre Paul qui les anticipait en apprenant aux Thessaloniciens à ne pas se plaindre "comme les autres qui n'ont pas d'espérance ". 